phys. stat. sol. (b) 161, 613 (1990)

Subject classification: $73.40 ; \mathrm{S} 8.15$

Sektion Physik der Humboldt-Universität zu Berlin ${ }^{1}$ )

Bereich Tieftemperatur-Festkörperphysik and Bereich Kristallographie

\title{
Magnetotransport Properties and Subband Structure of the Two-Dimensional Electron Gas in the Inversion Layer of $\mathrm{Hg}_{1-x} \mathrm{Cd}_{x} \mathrm{Te}$ Bicrystals
}

\author{
By \\ W. KraAK, J. Kaldasch, P. Gille, Th. Schurig, and R. HerrmanN
}

\begin{abstract}
The electronic and magnetotransport properties of conduction electrons in the grain boundary interface of p-type $\mathrm{Hg}_{1-x} \mathrm{Cd}_{x} \mathrm{Te}$ bicrystals are investigated. The results clearly demonstrate the existence of a two-dimensional degenerate n-type inversion layer in the vicinity of the grain boundary. The observed quantum oscillations of the magnetoresistivity result from a superposition of the Shubnikov-de Haas effect in several occupied electric subbands. The occupation of higher subbands is presumable depending on the total carrier density $n_{\mathrm{s}}$ of the inversion layer. Electron densities, subband energies, and effective masses of these electric subbands in samples with different total densities are determined. The effective masses of lower subbands are markedly different from the band edge values of the bulk material, their values decrease with decreasing electron density and converging to the bulk values at lower densities. This agrees with predictions of the triangular potential well model and a pronounced nonparabolicity of the energy bands in $\mathrm{Hg}_{1-x} \mathrm{Cd}_{x} \mathrm{Te}$. At high magnetic fields $(B>10 \mathrm{~T})$ it is experimentally verified that the Hall resistivity $\varrho_{x y}$ is quantized into integer multiplies of $h / e^{2}$.
\end{abstract}

Es werden die elektrischen und Magneto-Transporteigenschaften der Leitungselektronen in Korngrenzen von $\mathrm{p}-\mathrm{Hg}_{1-x} \mathrm{Cd}_{x} \mathrm{Te}$-Bikristallen untersucht. Die Ergebnisse zeigen eindeutig, daß in der unmittelbaren Umgebung der Korngrenzen eine entartete n-leitende Inversionsschicht existiert. Die beobachteten Quantenoszillationen des Magnetowiderstands resultieren aus der Überlagerung von Shubnikov-de Haas-Oszillationen in mehreren besetzten elektrischen Subbändern. Die Besetzung höherer Subbänder ist vorrangig von der Gesamtladungsträgerkonzentration in der Inversionsschicht abhängig. Durch die Auswertung der Quantenoszillationen des Magnetowiderstandes $\varrho_{x x}$ können die charakteristischen Parameter der elektronischen Subbandstruktur (Ladungsträgerdichten $n_{\text {si }}$, Subbandenergien $E_{\mathrm{F}}-E_{\mathrm{i}}$ und effektive Elektronenmassen $m_{\mathrm{ci}}^{*}$ ) ermittelt werden. Die effektiven Massen der unteren Subbänder unterscheiden sich deutlich von den Werten der Bandkantenmassen des Volumenmaterials; ihre Werte nehmen mit abnehmender Ladungsträgerdichte $a b$ und konvergieren zu den Volumendaten bei niedrigen Ladungsträgerdichten. Dieses Verhalten stimmt mit den Voraussagen eines einfachen theoretischen Modells unter Verwendung einer Dreieckpotentialtopf-Näherung und einer ausgeprägten Nichtparabolizität des Leitungsbandes gut überein. In hohen Magnetfeldern $(B>10 \mathrm{~T})$ werden experimentelle Bedingungen realisiert, bei denen der Hallwiderstand $\varrho_{x y}$ in ganzzahligen Vielfachen von $h / e^{2}$ quantisiert ist.

\section{Introduction}

The alloy system $\mathrm{Hg}_{1-x} \mathrm{Cd}_{x} \mathrm{Te}$ has been the subject of numerous investigations in recent years. Our interest in these studies stems from three related reasons. First, the alloys with $x=0.2$ to 0.3 have received a great amount of attention because of their application for long-wavelength infrared detectors and other electronic devices. The development of such

1) Invalidenstr. 110, DDR-1040 Berlin, GDR. 
devices requires an exact knowledge of the electrical and galvanomagnetic parameters of this material. The electrical properties of $\mathrm{n}$-type $\mathrm{Hg}_{1-x} \mathrm{Cd}_{x} \mathrm{Te}$ have been extensively studied and are understood in a qualitative manner. However, for p-type $\mathrm{Hg}_{1-x} \mathrm{Cd}_{x} \mathrm{Te}$ with carrier concentrations $p<10^{17} \mathrm{~cm}^{-3}$ the properties of polycrystalline samples show many anomalies in the electrical transport phenomena which are not directly related with the electronic band structure. In particular, pronounced dips and douple reversals of the sign of the Hall coefficient occur as the temperature is reduced (e.g. [1]). It was shown $([1,2]$ and references therein) that this anomalous behaviour may be produced by inversion or accumulation layers on the surface and/or various internal interfaces. The purpose of the present paper is to show that grain boundaries in the $\mathrm{HgCdTe}$ material can drastically alter and dominate the measured transport properties at low temperatures.

Secondly, the electronic properties of grain boundaries in semiconductors have attracted increasing interest over the past few years. Recently it was demonstrated by magnetotransport investigations of the electronic properties of grain boundaries in various semiconducting materials that the defects in the grain boundary region are evidently leading to the formation of a natural quantum well in which a degenerate carrier gas (inversion or accumulation layer) forms a quasi-two-dimensional system. In this system the carrier gas is confined in a symmetric V-like potential well which quantizes the motion of the carriers in the direction perpendicular to the grain boundary interface. For example, such inversion layers adjacent to grain boundaries are found in $\mathrm{n}-\mathrm{Ge}[3,4]$, p-InSb [5, 6], and $\mathrm{p}-\mathrm{Hg}_{1-x} \mathrm{Mn}_{x} \mathrm{Te}[7,8]$.

The interesting properties of this disordered quasi-two-dimensional electronic system have been demonstrated by the observation of characteristic quantum properties in this novel 2D-system including subband quantization, integral quantum Hall effect [8 to 11], and weak localization effects [12]. However, the nature of the interface states in the grain boundary is not yet completely clear. Dangling bonds and segregated impurities seem to be the most important defects in the vicinity of the grain boundary interface. Detailed studies of the electrical transport properties of grain boundaries may give valuable information about the individual electronic properties and the nature of defects at the interface and so are highly desirable.

Moreover, the narrow gap semiconductors (especially $\mathrm{InSb}$ and $\mathrm{Hg}_{1-x} \mathrm{Cd}_{x} \mathrm{Te}$ ) offer a unique band structure and combination of electronic properties (e.g. extremely low effective electron masses, high carrier mobilities, high effective $g$-factors, nonparabolicity of the energy bands, and a unique composition and pressure dependence of the energy band parameters). These properties result in large Landau level spacings and pronounced quantum effects in high magnetic fields. As a consequence, these alloy systems are excellent candidates for the investigations of quantum phenomena in reduced dimensions. Also from this point of view the study of the electronic and quantum properties of the natural $2 \mathrm{D}$ system adjacent to grain boundaries in $\mathrm{HgCdTe}$ bicrystals may be of great interest.

In this paper we present electrical and magnetotransport properties of the grain boundary interface in p-type $\mathrm{Hg}_{1-x} \mathrm{Cd}_{x} \mathrm{Te}$ bicrystals which indicate that a degenerate n-inversion layer exists adjacent to grain boundaries. By means of the quantum oscillations of the magnetoresistivity the characteristic parameters of the Q2DEG (subband population, effective electron masses, subband separation) were established. The obtained experimental data are in good agreement with simple calculations based on a triangular potential well approximation and taking into account a pronounced nonparabolicity of the energy bands in the narrow gap semiconductor $\mathrm{Hg}_{1-x} \mathrm{Cd}_{x} \mathrm{Te}$. The observation of the integral quantum 
Hall effect in the inversion layer of $\mathrm{HgCdTe}$ bicrystals demonstrates the universal twodimensional properties of the electron gas confined in the symmetrical potential well of the grain boundary.

\section{Experimental Details}

The crystals studied were grown in our laboratory by the seedless travelling heater method (THM) using Te as a solvent. THM is a crystal growth technique which combines the advantages of low temperature methods and steady-state growth known from zone melting. It has been proved to be especially suitable to the segregation problem connected with mixed crystals. Starting from $5 \mathrm{~N}$ elements combined with further purification efforts (destillation, zone melting) polycrystalline source materials have been homogenized from the binary components separately synthetized before. The crystals have been grown from a Te-rich solution at a temperature of $550^{\circ} \mathrm{C}$ with a growth rate of $1.0 \mathrm{~mm}$ per day.

A typical ingot consists of several differently oriented grains with volumes up to $1 \mathrm{~cm}^{3}$. The polycrystalline structure can be clearly seen after selective etching in a solution of $30 \%$ $\mathrm{HNO}_{3} ; 20 \% \mathrm{HCl}$, and $50 \% \mathrm{H}_{2} \mathrm{O}$ (Fig. 1). The mole fraction $x$ of our $\mathrm{Hg}_{1-x} \mathrm{Cd}_{x} \mathrm{Te}$ samples has been measured by electron probe microanalysis using $\mathrm{Cd}-\mathrm{L}_{\alpha}$ radiation.

$\mathrm{Hg}_{1-x} \mathrm{Cd}_{x} \mathrm{Te}$ bicrystal samples containing a single grain boundary are cut from the as-grown ingot by a wire saw into rectangular Hall bars with maximum dimensions of $1 \times 1 \times 5 \mathrm{~mm}^{3}$. After etching the samples (see above) to remove the damaged surface layer reliable electrical contacts were attached to the grain boundary plane. Electrical current contacts $(0.1 \mathrm{~mm} \mathrm{Au})$ were soldered to the sample, potential leads $(0.025 \mathrm{~mm} \mathrm{Au})$ were spark bonded by use of a capacitor discharge.

All measurements of the resistivity and the Hall constant of the sample were done with standard dc technique. The current fed to the sample was adjusted to levels where ohmic behaviour was guaranted. Shubnikov-de Haas $(\mathrm{SdH})$ oscillations have been recorded with

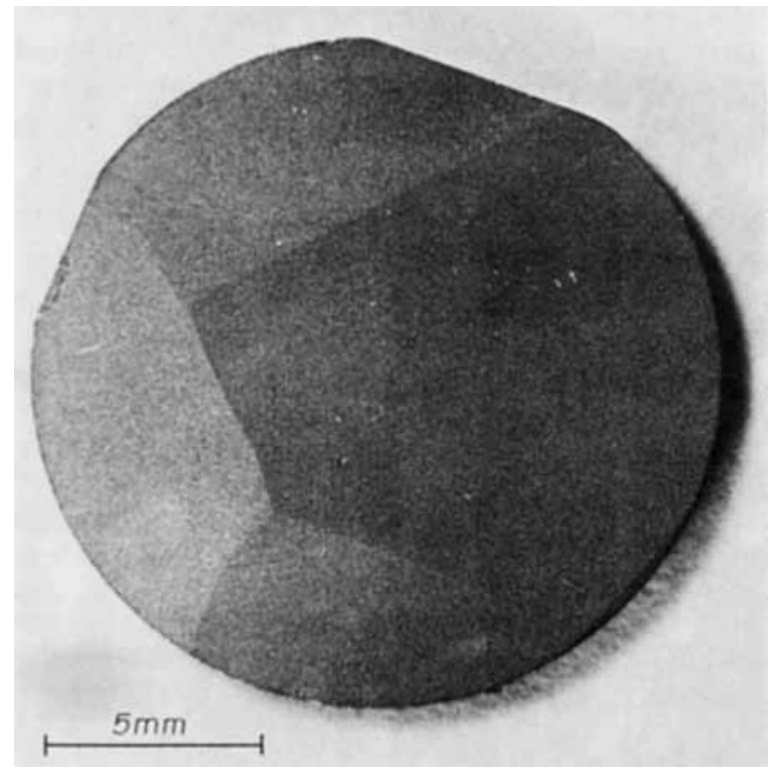

Fig. 1. Micrograph of an etched cross-section of a $\mathrm{Hg}_{1-x} \mathrm{Cd}_{x} \mathrm{Te}$ slice showing few differently oriented monocrystalline grains. From these slices bicrystal samples containing a single grain boundary were cut 
either dc technique or a sensitive ac technique using ac magnetic field modulation and phase sensitive detection techniques. The high field experiments $(B>5 \mathrm{~T})$ have been performed in a $15 \mathrm{~T}$ superconducting solenoid at the International Laboratory for High Magnetic Fields and Low Temperatures in Wroclaw (Poland).

\section{Experimental Results and Discussion}

\subsection{Evidence for inversion layers in the grain boundary region}

We have studied the magnetotransport properties of several bicrystal samples of p-type $\mathrm{Hg}_{1-x} \mathrm{Cd}_{x} \mathrm{Te}(0.2<x<0.3)$ containing a single grain boundary. For comparison, monocrystalline "control" samples cut closely to the grain boundary from the same ingot were used for an investigation of the bulk material properties. Hall measurements indicate that the bulk samples are p-type with a net acceptor concentration $N_{\mathrm{A}}-N_{\mathrm{D}} \approx 10^{15} \mathrm{~cm}^{-3}$; this places the Fermi level in the bulk material at $\approx 10 \mathrm{meV}$ above the top of the valence band.

Striking differences have been found for the electrical and galvanomagnetic properties of the monocrystalline and bicrystal samples. Fig. 2 shows typical temperature dependences of the electrical resistivity $\varrho$ in the range from 4.2 to $300 \mathrm{~K}$ of a monocrystalline sample $(x \approx 0.2)$ and a sample containing the grain boundary. The bulk resistivity at low temperatures $(T<12 \mathrm{~K})$ is almost one order of magnitude higher than the boundary resistivity and shows a temperature dependence typical for narrow gap semiconductors: a degenerate behaviour at low temperatures and a thermally activated one at higher temperatures (a direct energy gap $E_{\mathrm{g}} \approx 50$ to $60 \mathrm{meV}$ has been determined for the sample with $x \approx 0.2$ ). In contrast to the semiconducting behaviour of the bulk material the bicrystal samples revealed a metallic-like temperature dependence in the whole temperature range investigated.

Furthermore, the sign of the Hall coefficient in all samples containing a grain boundary has been found negative $\left(R_{\mathrm{H}}<0\right)$ and to be temperature independent in the whole temperature range. On the contrary, the galvanomagnetic properties of the bulk material samples show a series of anomalies consisting of single or double reversals of the sign of the Hall coefficient going, for example, from negative $\left(R_{\mathrm{H}}<0\right)$ to positive $\left(R_{\mathrm{H}}>0\right)$ and back to negative values $\left(R_{\mathrm{H}}<0\right)$ as the temperature is reduced from 300 to $4.2 \mathrm{~K}$. Similar

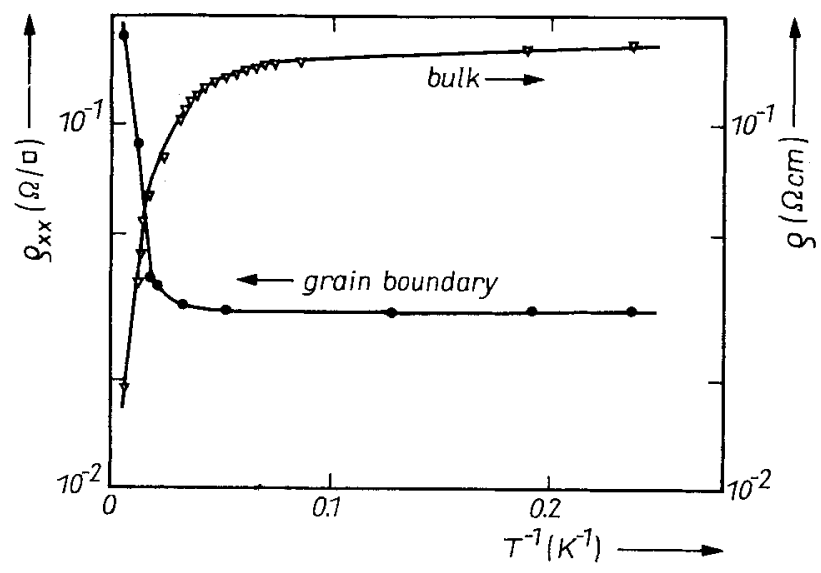

Fig. 2. Electrical resistivity as a function of reciprocal temperature for a p-type $\mathrm{Hg}_{1-x} \mathrm{Cd}_{x} \mathrm{Te}$ monocrystal (sample $\mathrm{TH} 256$ ) and an adjacent bicrystal sample containing a single grain boundary 


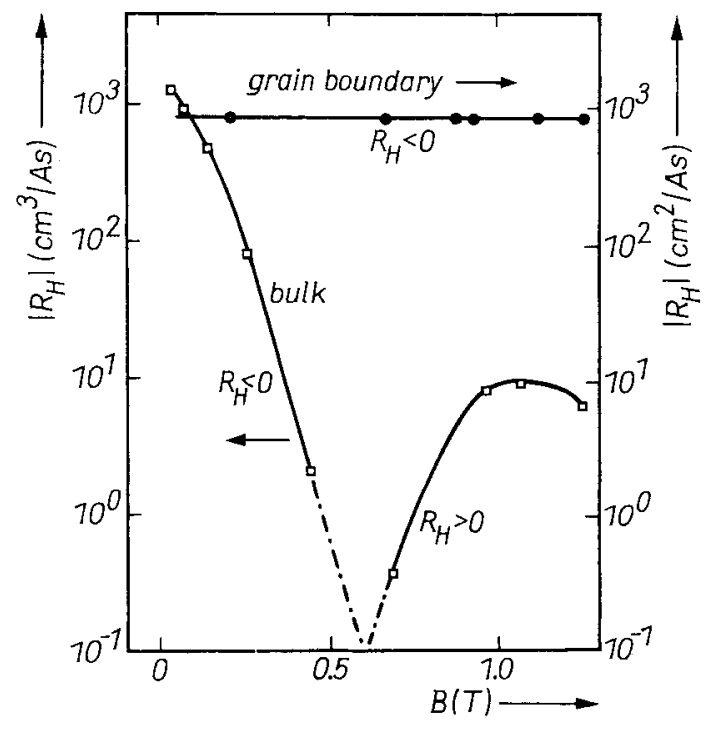

Fig. 3. Magnetic field dependence of the Hall coefficient $R_{\mathrm{H}}$ for a monocrystal and a bicrystal sample (TH256); $T=4.2 \mathrm{~K}$

differences have been found for the magnetic field dependences of the Hall coefficient (Fig. 3). Whereas for the samples with grain boundaries the magnitude of $R_{\mathrm{H}}\left(R_{\mathrm{H}}<0\right)$ is practically independent or weakly dependent on the magnetic field, for the p-type bulk material sample a sign reversal of the Hall coefficient is evident in this picture: in weak magnetic fields $(B<0.6 \mathrm{~T}) R_{\mathrm{H}}$ is negative $\left(R_{\mathrm{H}}<0\right)$ and as the magnetic field increases $R_{\mathrm{H}}$ changes sign $\left(R_{\mathrm{H}}>0\right)$ and reaches a saturation value for $B>1 \mathrm{~T}$. Such anomalies of the galvanomagnetic properties of p-type bulk samples of the alloy system $\mathrm{Hg}_{1-x} \mathrm{Cd}_{x} \mathrm{Te}(x<0.3)$ have been observed by many researchers and a variety of explanations have been proposed. The results of these experiments indicate that the anomalous electrical and galvanomagnetic properties at low temperatures in $\mathrm{p}-\mathrm{Hg}_{1-x} \mathrm{Cd}_{x} \mathrm{Te}$ are mainly due to an inversion layer at the surface of the sample (e.g. $[1,2]$ and references therein).

Finally, pronounced quantum oscillation of the magnetoresistivity $\varrho_{x x}$ have been observed in the bicrystal samples due to the quasi-two-dimensional properties of the $\mathrm{n}$-inversion layer in the grain boundary interface (cf. also Section 3.2). On the contrary, for the bulk material no $\mathrm{SdH}$ oscillations and a almost steady increase of the resistivity in magnetic fields up to $5 \mathrm{~T}$ were found.

The striking differences between the galvanomagnetic properties of the bulk and bicrystal samples found in our experiments clearly demonstrate that a strongly degenerate n-type inversion layer must exist in the vicinity of the grain boundary. The much more pronounced and very stable degenerate electrical properties of the bicrystal samples (no evidence for any change of these properties by different sample preparation conditions has been found by our experiments) indicate that the charge trapping of defects at the grain boundary interface and their influence on the electronic properties must be considerably more effective than the defects at the surface inversion layer.

\subsection{Quantum properties of the $2 D E G$ in the inversion layer}

The Shubnikov-de Haas effect has proved to be an important tool for characterizing the properties and electronic structure of semiconductors (carrier concentration, effective masses, $g$-factors, Dingle temperature, etc.) both in 3D and 2D systems. For the investigation of the two-dimensional electronic system in the grain boundary quantum well low-temperature magnetoresistance measurements were made with the plane of the $2 \mathrm{DEG}$ in the inversion layer perpendicular and parallel with respect to the applied magnetic field. 
In the perpendicular orientation we have investigated several $\mathrm{Hg}_{1-x} \mathrm{Cd}_{x} \mathrm{Te}$ bicrystal samples, for the majority of which besides the anomalies mentioned above $\left(R_{\mathrm{H}}<0\right.$, degenerate behaviour of $\varrho$ and $R_{\mathrm{H}}$, cf. Section 3.1) very pronounced $\mathrm{SdH}$ oscillations were observed corresponding to Landau quantization of the electron gas in the inversion layer. Fig. 4 and 5 show typical low-temperature $\mathrm{SdH}$ plots of the magnetoresistance component $\varrho_{x x}(B)$ for two $\mathrm{Hg}_{1-x} \mathrm{Cd}_{x} \mathrm{Te}$ bicrystals taken from different ingots (TH 364, TH 256) with different parameters of the electronic structure of the bulk material.

As in the case of the inversion layer of $\operatorname{InSb}$ bicrystals $[5,6]$ these investigations show that the electron gas of the inversion layer in $\mathrm{Hg}_{1-x} \mathrm{Cd}_{x} \mathrm{Te}$ grain boundaries is confined in a potential well with a scale of length such that quantum effects are important $\left(d<\lambda_{\mathrm{B}}\right.$, where $\lambda_{\mathrm{B}}$ is the electron wavelength). In particular, the confinement of carriers normal to the interface in the symmetric electrostatic potential gives rise to a set of electric subbands $E_{i}+\hbar^{2} k_{\|}^{2} / 2 m^{*}(i=0,1,2)$.

As in InSb bicrystals the small effective electron mass and the small 2D density of states in $\mathrm{Hg}_{1-x} \mathrm{Cd}_{x} \mathrm{Te}$ leads to the filling of several electric subbands. The application of a magnetic field perpendicular to the interface causes additional quantization. The system becomes fully quantized with energy levels given by

$$
E_{i, n, s}=E_{i}+\left(n+\frac{1}{2}\right) \hbar \omega_{\mathrm{c}}+s g \mu_{\mathbf{B}} B
$$

where $E_{i}$ is the bottom of the $i$-th subband caused by the electric quantization in the direction normal to the interface; the second and third terms are the magnetic field dependent

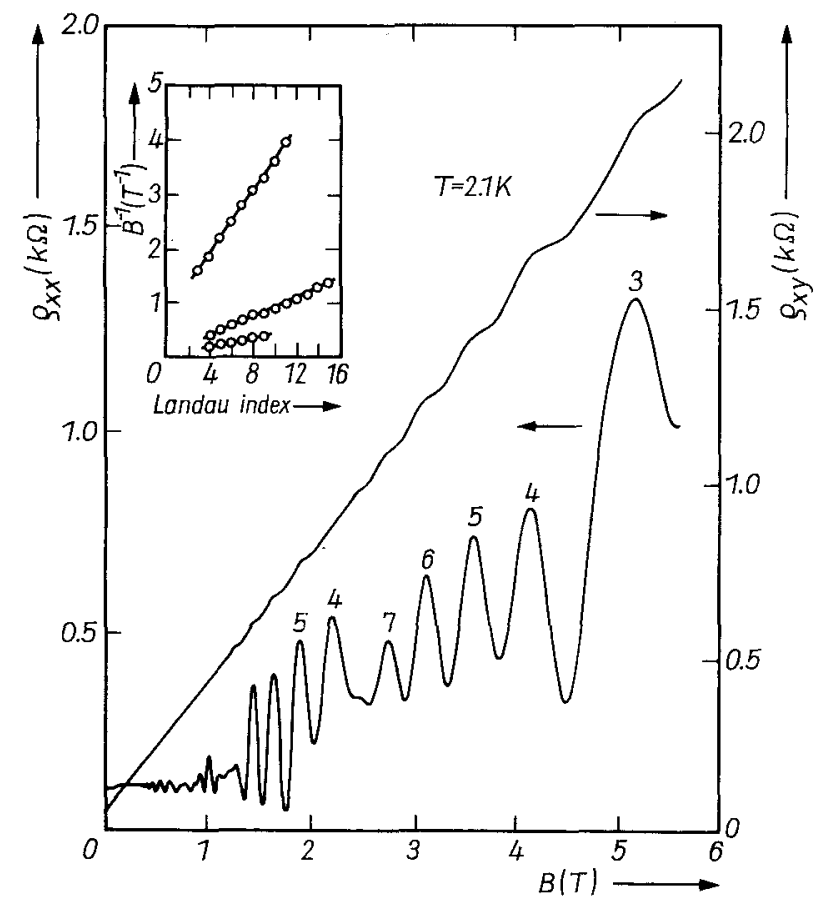

Fig. 4. Typical SdH oscillations of the resistivity $\varrho_{x x}$ of an n-inversion layer adjacent to the grain boundary in $\mathrm{p}-\mathrm{Hg}_{1-x} \mathrm{Cd}_{x} \mathrm{Te}$ bicrystals (sample TH 364/16). The index $l$ is the Landau level index of the corresponding resistivity peak. The field dependence of the Hall resistivity $\varrho_{x y}$ is also shown. The inset shows a standard plot of the inverse of the field positions of the extrema vs. Landau level index for the $i=0$ (lowest line), 1, and 2 (upper line) subbands 


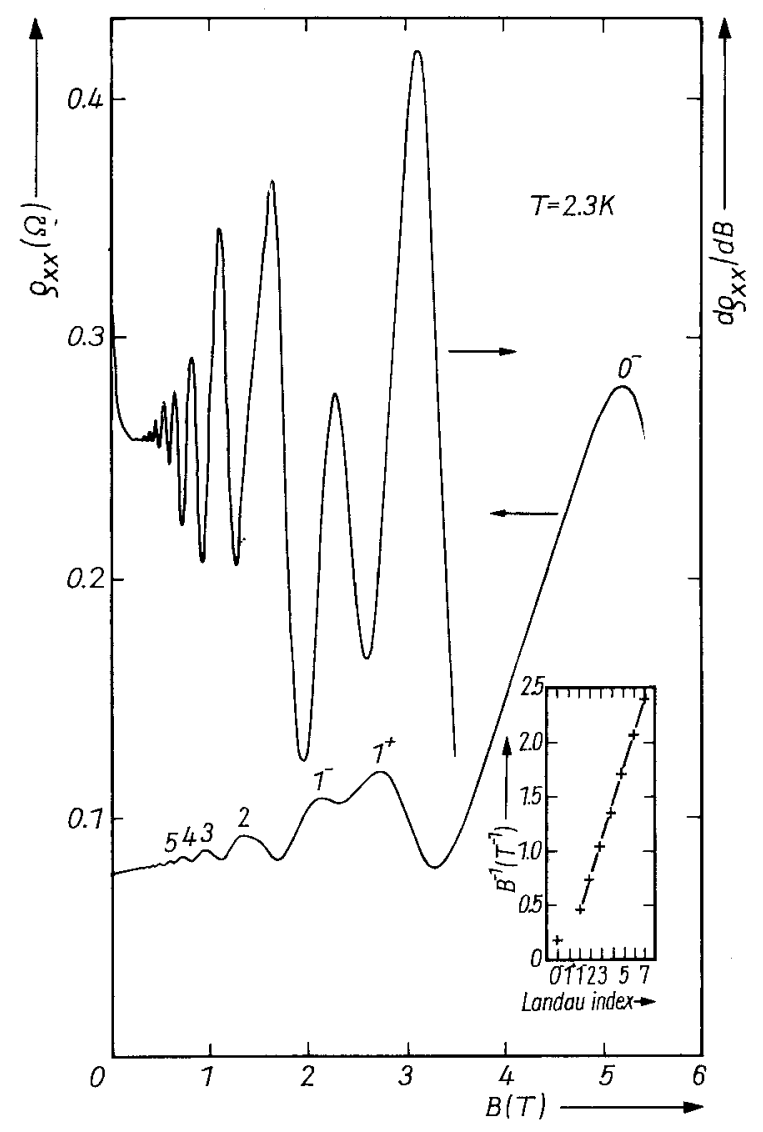

Fig. 5. Typical SdH oscillation of the resistivity component $\varrho_{x x}$ and the first derivation $\mathrm{d} \varrho_{x x} / \mathrm{d} B$ for bicrystal sample TH 256 of $\mathrm{Hg}_{1-x} \mathrm{Cd}_{x} \mathrm{Te}(x=0.2)$. The inset shows a standard $\mathrm{SdH}-$ plot $1 / B$ vs. Landau level index. In high magnetic field spin splitting of the Landau level $(n=1)$ is observed

Landau levels and spin splitting energies, respectively; $\omega_{\mathrm{c}}=e B / m^{*}$ is the cyclotron frequency, $s= \pm 1 / 2$ the spin quantum number, $g$ the Landê factor, and $\mu_{\mathrm{B}}$ the Bohr magneton. For every electric subband a ladder of Landau levels $l=0,1,2 \ldots$ exists. A sweep of the magnetic field leads to a superposition of the quantum oscillations for each subband, so that, if the oscillation period from each subband can be separated, the properties of each subband can be determined. Maxima in the density of states (and of the resistivity component $\left.\varrho_{x x}\right)$ occur if the Fermi energy $E_{\mathrm{F}}=\left(n+\frac{1}{2}\right) \hbar \omega_{\mathrm{c}}$. From the field position of the maxima of $\varrho_{x x}$ one can construct a standard diagram of $1 / B$ versus Landau level index (insets of Fig. 4 and 5). For the 2D case the frequency of each set of oscillations determines the carrier density in the subband $n_{\mathrm{si}}$ using the relation for an isotropic $2 \mathrm{DEG}$,

$$
n_{\mathrm{si}}=\frac{e}{\pi \hbar \Delta_{i}(1 / B)}
$$

where $A(1 / B)$ is the oscillation period in the reciprocal magnetic field, $e$ and $h$ are electron charge and Planck constant, respectively.

For all bicrystal samples of the ingot TH364 $(x \approx 0.23)$ the $\mathrm{SdH}$ oscillations (Fig. 4) show at least two, however normally three, distinct oscillation periods, which clearly reveal 
the occupation of several electric subbands. Because of the complex structure of the SdH spectra the data analysis was accomplished using Fourier analysis methods, which reveals the dominant frequency components. In accordance with the simple standard analysis of the SdH data (Fig. 4) the Fourier spectrum shows that three periods in reciprocal magnetic field can be identified. The total electron density calculated from the $\mathrm{SdH}$ periods $n_{\mathrm{s}}=\sum n_{\mathrm{si}} \approx 2.0 \times 10^{12} \mathrm{~cm}^{-2}$ is in good agreement with the carrier concentration determined from the classical Hall effect which is given by the relationship $n_{\mathrm{s}}=1 / e R_{\mathrm{H}}$ in the limit $\omega_{\mathrm{c}} \tau \gg 1$.

The occupation of the higher electric subbands presumable depends on the total carrier density $n_{\mathrm{s}}$ of the inversion layer. This is clearly shown by the results summarized in Table 1, where the occupation of different subbands for inversion layers with different total electron concentrations is demonstrated.

The occupation of several electric subbands is a consequence of the small effective electron mass $m^{*}$ and the low 2D density of states $D(E)=m^{*} / \pi \hbar^{2}[13]$ in narrow gap semiconductors.

From the $\mathrm{SdH}$ data we have determined the partial occupancies $n_{\mathrm{si}} / n_{\mathrm{s}}$ which are $0.59 n_{\mathrm{s}}$, $0.29 n_{\mathrm{s}}$, and $0.124 n_{\mathrm{s}}$ for the first three subbands $i=1,2$, and 3 , respectively. These values are very close to corresponding experimental values reported by Scholz [13] and Zao [14] for inversion layers in $\mathrm{Hg}_{1-x} \mathrm{Cd}_{x} \mathrm{Te}$ MIS structures and by Sobkowicz et al. [15] for $\mathrm{HgCdMnTe}$ bicrystals of material with similar alloy composition (see also Table 4).

The onset of the oscillation (sample Th 364/16) with the lowest frequency (for the subband $i=2$ ) was observable at magnetic fields $B \leqq 0.25 \mathrm{~T}$ indicating a high mobility $\mu \approx 4.87 \times 10^{4} \mathrm{~cm}^{2} / \mathrm{Vs}$ of these carriers. The onset of observable oscillations for the lower subbands occurs at correspondingly higher magnetic fields (Fig. 4) indicative of lower mobilities for these carriers. These results are consistent with the fact that the carriers in the lowest electric subband $(i=0)$ are confined closer to the grain boundary interface (see also Fig. 6) and feel the defect scattering at the interface more strongly. Moreover, the carriers in the lowest subband can screen the electric field of the scattering centres reducing their influence on the carriers of higher subbands.

In order to check the effective mass values of the inversion electrons we have carried out an analysis of the temperature dependence of the oscillation amplitudes $A(T)$. Such an analysis is favoured by the excellent $\mathrm{SdH}$ oscillations observed for the direct resistivity component $\varrho_{x x}$ (no modulation technique was required to detect the pronounced quantum oscillation $\varrho_{x x}(B)$ for the three subbands). Nevertheless, the results should be interpreted with some caution because of the complex oscillatory structure of the $\mathrm{SdH}$ patterns. The analysis of the temperature dependence of the oscillation was used to deduce the effective cyclotron masses for each subband.

Table 1

Subband occupation versus total inversion density $n_{\mathrm{s}}$ for different $\mathrm{Hg}_{1-x} \mathrm{Cd}_{x} \mathrm{Te}$ bicrystals

\begin{tabular}{lll}
\hline sample & $\begin{array}{l}n_{\mathrm{s}}=\sum n_{\mathrm{si}} \\
\left(\mathrm{cm}^{-2}\right)\end{array}$ & $\begin{array}{l}\text { number of identified } \\
\text { electric subbands }\end{array}$ \\
\hline TH256 $\quad(x \approx 0.2)$ & $1.4 \times 10^{11}$ & 1 \\
TH364/12 $(x \approx 0.26)$ & $1.43 \times 10^{12}$ & 2 \\
TH 364/16 $(x \approx 0.23)$ & $2.0 \times 10^{12}$ & 3 \\
\hline
\end{tabular}




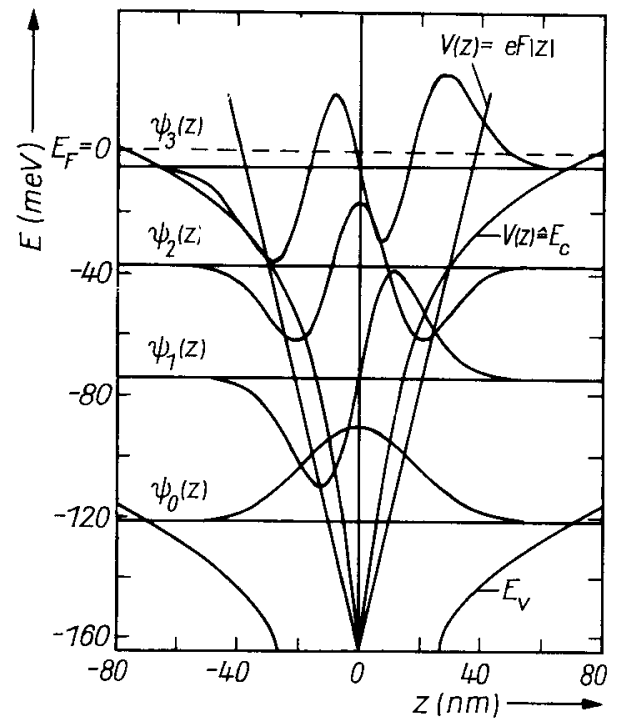

Fig. 6. Self-consistently calculated potential shapes of the bottom of the conduction band $E_{\mathrm{c}}$ and the top of the valence band $E_{\mathrm{v}}$ and the approximated symmetrical triangular potential $V(z)=-e F|z|$ of the grain boundary interface region using a total inversion density $n_{\mathrm{s}}$ $=2 \times 10^{12} \mathrm{~cm}^{-2}$. The calculated subband energy levels $E_{i}$ and the wave functions $\psi_{i}(z)$ for each subband are shown
Due to the strongly non-parabolic character of the conduction band in $\mathrm{Hg}_{1-x} \mathrm{Cd}_{x} \mathrm{Te}$ the cyclotron masses $m_{\mathrm{ci}}^{*}$ are different for different electric subbands. The effective cyclotron mass of the lowest subband is the highest $\left(\approx 0.037 m_{0}\right)$ and deviates considerably from the bulk values for this composition $\left(\approx 0.0115 m_{0}\right)$. The higher subbands

exhibit considerably smaller effective masses compared with the $i=0$ subband. The measured effective masses, which decrease with the subband number, are comparable with the calculated values. The results are summarized in Table 2.

All these features mentioned above (occupation of several electric subbands, strong energy dependence of the cyclotron masses $m_{\mathrm{ci}}^{*}$ ) are characteristic features of $2 \mathrm{D}$ structures involving narrow gap semiconductors.

The pronounced quantum oscillations $\varrho_{x x}(B)$ observed at low temperatures in the inversion layer of the grain boundary interface disappear when the magnetic field is applied parallel to the interface and are replaced by long period oscillations in the derivative of the magnetoresistance that were not periodic in inverse magnetic field. This strong field orientation dependence gives a further convincing evidence for the $2 \mathrm{D}$ character of the 2DEG in the inversion layer. The observed special structure of field dependences in a parallel magnetic field can be explained by a subsequent depopulation of the electric subbands. These results will be reported elsewhere.

For all bicrystal samples of the ingot TH256 $(x=0.2)$ only one single oscillation period was observed indicating the occupation of the lowest electric subband only (Fig. 5). A surprisingly small electron density $n_{\mathrm{s} 0}=(1.4 \pm 0.1) \times 10^{11} \mathrm{~cm}^{-2}$ has been derived from the $\mathrm{SdH}$ oscillation data. This value is much lower than the total carrier concentration derived from the Hall coefficient $R_{\mathrm{H}}$ (cf. Table 3). This striking discrepancy suggests that the conductivity of the $\mathrm{HgCdTe}$ bulk material may be not negligible compared with the quantum well and therefore the classical transport properties will be strongly affected by parallel conduction.

The onset of observable oscillations for the lowest subband occurs at magnetic fields $B \approx 0.25 \mathrm{~T}$ indicating a high carrier mobility $\mu_{0}=4.76 \times 10^{4}\left(\mathrm{~cm}^{2} / \mathrm{Vs}\right)$ for the inversion electrons in the lowest subband $i=0$. In high magnetic fields $(B>2 \mathrm{~T})$ pronounced spin splitting is clearly evident for the Landau level $n=1$ (cf. Fig. 5). An effective $g$-Landé factor of -113 has been derived from the results (cf. Table 3). The subband parameters of the 
Table 2

Experimental and theoretical parameters of the 2DEG subband structure in an $\mathrm{Hg}_{1-x} \mathrm{Cd}_{x} \mathrm{Te}$ inversion layer (sample $364 / 16, n_{\mathrm{s}}=2.0 \times 10^{12} \mathrm{~cm}^{-2}$ )

\begin{tabular}{|c|c|c|c|}
\hline \multirow[t]{2}{*}{ parameter } & \multicolumn{2}{|l|}{ experiment } & \multirow{2}{*}{$\begin{array}{l}\text { theory } \\
\text { triangular } \\
\text { potential }\end{array}$} \\
\hline & ordinary $\mathrm{SdH}$ plot & Fourier transform & \\
\hline $\begin{array}{l}n_{0}\left(\mathrm{~cm}^{-2}\right) \\
n_{1}\left(\mathrm{~cm}^{-2}\right) \\
n_{2}\left(\mathrm{~cm}^{-2}\right) \\
n_{3}\left(\mathrm{~cm}^{-2}\right)\end{array}$ & $\begin{array}{l}(1.07 \pm 0.01) \times 10^{12} \\
(3.9 \pm 0.1) \times 10^{11} \\
(1.5 \pm 0.2) \times 10^{11} \\
-\end{array}$ & $\begin{array}{l}1.2 \times 10^{12} \\
5.9 \times 10^{11} \\
2.35 \times 10^{11} \\
-\end{array}$ & $\begin{array}{l}1.17 \times 10^{12} \\
5.7 \times 10^{11} \\
2.3 \times 10^{11} \\
5 \times 10^{10}\end{array}$ \\
\hline$n_{\mathrm{s}}=\sum n_{\mathrm{si}}\left(\mathrm{cm}^{-2}\right)$ & $1.6 \times 10^{12}$ & $2.02 \times 10^{12}$ & $2.02 \times 10^{12}$ \\
\hline $\begin{array}{l}m_{\mathrm{c} 0} / m_{0} \\
m_{\mathrm{c} 1} / m_{0} \\
m_{\mathrm{c} 2} / m_{0} \\
m_{\mathrm{c} 3} / m_{0}\end{array}$ & $\begin{array}{l}0.037 \pm 0.008 \\
0.023 \pm 0.003 \\
0.017 \pm 0.002 \\
-\end{array}$ & & $\begin{array}{l}0.035 \\
0.025 \\
0.018 \\
0.012\end{array}$ \\
\hline$E_{\mathbf{F}}-E_{0}$ & $\begin{cases}0.105 & (*) \\
0.077 & (* *)\end{cases}$ & & 0.120 \\
\hline$E_{\mathrm{F}}-E_{1}$ & $\begin{cases}0.063 & (*) \\
0.061 & (* *)\end{cases}$ & & 0.073 \\
\hline$E_{\mathbf{F}}-E_{2}$ & $\begin{cases}0.030 & (*) \\
0.031 & (* *)\end{cases}$ & & 0.037 \\
\hline$E_{\mathbf{F}}-E_{3}$ & & & 0.005 \\
\hline$n_{\mathrm{H}}=1 / R_{\mathrm{H}}\left(\mathrm{cm}^{-2}\right)$ & $(2.03 \pm 0.5) \times 10^{12}$ & & \\
\hline $\begin{array}{l}\mu_{4.2 \mathrm{~K}}\left(\mathrm{~cm}^{2} / \mathrm{Vs}\right) \\
\mu_{2.2 \mathrm{~K}}\left(\mathrm{~cm}^{2} / \mathrm{Vs}\right) \\
\mu_{4.2 \mathrm{~K}}=\sigma R_{\mathrm{H}} \\
\left(\mathrm{cm}^{2} / \mathrm{Vs}\right)\end{array}$ & $\begin{array}{l}4.0 \times 10^{4} \quad(* * *) \\
4.87 \times 10^{4} \quad(* * *) \\
4.4 \times 10^{4} \quad(* * * *)\end{array}$ & & \\
\hline
\end{tabular}

Remarks: Material parameters used for the calculations: $x=0.23, E_{\mathbf{g}}=118 \mathrm{meV}$, $m_{\mathrm{n}}=0.0115 m_{0}[16], N_{\mathrm{A}}-N_{\mathrm{D}}=1.0 \times 10^{15} \mathrm{~cm}^{-3}, \varepsilon_{\mathrm{F}}=10 \mathrm{meV}$.

The values of the cyclotron masses at the Fermi level of the corresponding subbands $m_{\mathrm{c} i} / m_{0}$ have been determined from the temperature dependence of the amplitude of SdH oscillations.

Using the values $m_{\mathrm{c} i}^{*} / m_{0}$ the subband energies $E_{\mathrm{F}}-E_{i}$ were determined in a parabolic approximation $(* *)$ from the relation

$$
E_{\mathrm{F}}-E_{i}=\frac{e \hbar}{m_{\mathrm{s} i}^{*} \Delta_{i}(1 / B)}=\frac{\hbar^{2} n_{\mathrm{si}} \pi}{m_{\mathrm{c} i}^{*}} .
$$

The values $\left(E_{\mathrm{F}}-E_{i}\right)(*)$ have been calculated with the use of Kane's nonparabolic dispersion relation [17],

$$
E_{\mathrm{F}}-E_{i}=-\frac{E_{\mathrm{g}}}{2} \pm\left\{\frac{E_{\mathrm{g}}^{2}}{2}+\frac{\hbar^{2} k_{\mathrm{F} i}^{2}}{2 m_{\mathrm{n}}^{*}} E_{\mathrm{g}}\right\}^{1 / 2}, \quad k_{\mathrm{F} i}=\left(2 \pi n_{\mathrm{s} i}\right)^{1 / 2} .
$$

The use of the bulk effective mass $m_{\mathrm{n}}$ in the grain boundary inversion layer is justified, because the interface potential spreads over many cells (cf. also Fig. 6). It should be noted that in comparison with the values obtained from the first method the latter values (*) (nonparabolic approximation) should be more reliable.

The carrier mobilities $\mu$ have been calculated from the oscillation onset $(\mu B \approx 1$ ) of the subband $i=2(* * *)$ or from the electrical transport measurements using the relation $\mu=\sigma R_{\mathrm{H}}(* * * *)$. 
2DEG in the inversion layer of sample TH256 determined from the experiments and the results of self-consistent calculations using a linear trinangular potential are summarized in Table 3.

In order to get some theoretical insight into the energy spectrum of the inversion layer some simple calculations were performed. Basing on previous subband calculations $[5,18]$, employing a numerical integration of the Poisson equation and a further semiclassical quantization of the potential approximated by a symmetric well $V(z)=e F|z|$ (with $F$ the electric field strength on both sides of the grain boundary plane as a fitting parameter) the fundamental subband parameters of the $2 \mathrm{DEG}$ in the inversion layer (subband population, effective masses, and subband energies) were calculated. The nonparabolicity of the conduction band has been taken into account using Kane's nonparabolic dispersion relation [17]. Other material parameters were taken from [16].

The detailed subband parameters of the $2 D E G$ in the inversion layer of the samples TH 364 and TH256 obtained from an analysis of the quantum oscillations at atmospheric pressure and the result of the self-consistent calculations are summarized in Tables 2 and 3 . A good agreement between the experimental and theoretical values has been found. The results of self-consistent calculations give some evidence for the occupation of a fourth

\section{Table 3}

Experimental and theoretical parameters of the 2DEG subband structure in an $\mathrm{Hg}_{1-x} \mathrm{Cd}_{x} \mathrm{Te}$ inversion layer (sample TH256, $n_{\mathrm{s}}=1.4 \times 10^{11} \mathrm{~cm}^{-2}$ )

\begin{tabular}{|c|c|c|c|}
\hline \multirow[t]{2}{*}{ parameter } & \multicolumn{2}{|l|}{ experiment } & \multirow{2}{*}{$\begin{array}{l}\text { theory } \\
\text { triangular } \\
\text { potential }\end{array}$} \\
\hline & standard SdH plot & Fourier transform & \\
\hline $\begin{array}{l}n_{0}\left(\mathrm{~cm}^{-2}\right) \\
n_{1}\left(\mathrm{~cm}^{-2}\right)\end{array}$ & $\begin{array}{l}(1.4 \pm 0.1) \times 10^{11} \\
-\end{array}$ & $\begin{array}{l}1.39 \times 10^{11} \\
-\end{array}$ & $\begin{array}{l}1.33 \times 10^{11} \\
0.07 \times 10^{11}\end{array}$ \\
\hline$n_{\mathrm{s}}\left(\mathrm{cm}^{-2}\right)$ & $1.4 \times 10^{11}$ & $1.39 \times 10^{11}$ & $1.4 \times 10^{11}$ \\
\hline $\begin{array}{l}m_{\mathrm{c} 0} / m_{0} \\
m_{\mathrm{c} 1} / m_{0}\end{array}$ & $\begin{array}{l}0.013 \pm 0.002 \\
-\end{array}$ & & $\begin{array}{l}0.012 \\
0.0067\end{array}$ \\
\hline $\begin{array}{l}E_{\mathrm{F}}-E_{0}(\mathrm{eV}) \\
E_{\mathrm{F}}-E_{1}(\mathrm{eV})\end{array}$ & $\begin{array}{l} \begin{cases}0.036 \pm 0.003 & (*) \\
0.0025 & (* *)\end{cases} \\
-\end{array}$ & & $\begin{array}{l}0.0343 \\
0.028\end{array}$ \\
\hline$n_{\mathrm{H}}=\frac{1}{e R_{\mathrm{H}}}\left(\mathrm{cm}^{-2}\right)$ & $(3.3 \pm 0.6) \times 10^{15}$ & & \\
\hline$g$ & $-(115 \pm 40)$ & & 83.3 \\
\hline$\mu\left(\mathrm{cm}^{2} / \mathrm{Vs}\right)$ & $4.76 \times 10^{4}$ & & \\
\hline
\end{tabular}

Remarks: cf. Table 2.

Material parameters used for the calculation: $x=0.2, E_{\mathrm{g}}=75 \mathrm{meV}, m_{\mathrm{n}}=0.0063[16]$, $N_{\mathrm{A}}-N_{\mathrm{D}}=1 \times 10^{15} \mathrm{~cm}^{-3}$. 
subband $(i=3$ ) (sample TH364/16) and a secound subband ( $i=1$ for sample TH 256). However, the occupation of these subbands is actually too small for experimental detection (even the application of a sensitive modulation technique gives no indication of such a subband). The good agreement of experimental and calculated results shows that the used calculation method is a sufficiently accurate approximation for the subband structure of the inversion layer. Similar results have been obtained previously for the $2 \mathrm{DEG}$ in the inversion layer of InSb bicrystals [5].

The Q2DEG of inversion and accumulation layers of MIS structures in $\mathrm{Hg}_{1-x} \mathrm{Cd}_{x} \mathrm{Te}$ has been the subject of intensive experimental [13, 14, 19 to 22] and theoretical [23, 24] investigations in the last few years. As in our case the fourth $(i=3)$ subband, although predicted by theory [23] for high total electron densities, was not observed till now, probably due to the experimental difficulties to measure such low carrier densities. Taking into account the nonparabolicity of the conduction band the band bending, the carrier concentration, and the complete 2D subband structure have been calculated. The results are discussed for a wide range of the total electron density $n_{\mathrm{s}}$ in the inversion layer. The calculation indicates the existence of three or four electric subbands for similar alloy compositions and $n_{\mathrm{s}}>1 \times 10^{12} \mathrm{~cm}^{-2}$. These latter results has not been confirmed by our

Table 4

Comparison of subband parameters obtained for inversion layers in $\mathrm{Hg}_{1-x} \mathrm{Cd}_{x} \mathrm{Te}$ bicrystals, $\mathrm{Hg}_{1-x} \mathrm{Cd}_{x} \mathrm{Te}$ MIS structures, and InSb bicrystals for similar compositions and interface charges

\begin{tabular}{lllll}
\hline & $\begin{array}{l}\text { subband } \\
\text { index } \\
i\end{array}$ & $\begin{array}{l}\text { subband } \\
\text { population } \\
n_{\mathrm{si}} / n_{\mathrm{s}}\end{array}$ & $\begin{array}{l}\text { effective } \\
\text { electron } \\
\text { masses } \\
m_{\mathrm{c} i}^{*} / m_{0}\end{array}$ & $\begin{array}{l}\text { subband } \\
\text { energies } \\
E_{\mathrm{F}}-E_{i}(\mathrm{eV})\end{array}$ \\
\hline present paper & 0 & 0.58 & 0.035 & 0.120 \\
(theory) & 1 & 0.28 & 0.025 & 0.073 \\
$n_{\mathrm{s}}=2 \times 10^{12} \mathrm{~cm}^{-2} ;$ & 2 & 0.13 & 0.018 & 0.037 \\
grain boundary & 3 & 0.01 & 0.012 & 0.005 \\
Scholz [13] & 0 & 0.6 & & \\
(experiment) & 1 & 0.29 & & \\
$n_{\mathrm{s}}>2 \times 10^{12} \mathrm{~cm}^{-2} ;$ & 2 & 0.10 & & \\
MIS-structure & 3 & - & & 0.122 \\
Zöllner [24] & 0 & 0.71 & 0.038 & 0.050 \\
(theory) & 1 & 0.22 & 0.0258 & \\
$n_{\mathrm{s}}=2 \times 10^{12} \mathrm{~cm}^{-2} ;$ & 2 & 0.06 & 0.015 & 0.0136 \\
MIS-structure & & & & 0.107 \\
Takada [23] & 0 & 0.72 & 0.039 & 0.051 \\
(theory) & 1 & 0.23 & 0.028 & \\
$n_{\mathrm{s}}=2 \times 10^{12} \mathrm{~cm}^{-2} ;$ & 2 & 0.05 & 0.019 & 0.023 \\
$\begin{array}{l}\text { Herrmann [25] } \\
\text { (experiment) }\end{array}$ & 0 & 0.67 & 0.027 & 0.123 \\
$n_{\mathrm{s}}=1.6 \times 10^{12} \mathrm{~cm}^{-2} ;$ & 2 & 0.22 & 0.021 & 0.026 \\
InSb grain boundary & 3 & 0.02 & - & 0.007 \\
\hline
\end{tabular}


experiments for the grain boundary interface, where for $n_{\mathrm{s}}=1.43 \times 10^{12} \mathrm{~cm}^{-2}$ only one electric subband is seen (cf. Table 1).

As seen from Table 3 the observed occupation ratio $n_{1} / n_{0}$ for the $\mathrm{Hg}_{1-x} \mathrm{Cd}_{x} \mathrm{Te}$ structures is anomalously high $\left(n_{1} / n_{0}<0.5\right)$ whereas in the similar system of InSb grain boundaries $n_{1} / n_{0} \approx 0.30$. Similar results have been obtained in $\mathrm{HgCdMnTe}$ grain boundaries and explained by the considerably smaller energy separation between the $i=0$ and 1 electric subbands in the $\mathrm{Hg}_{1-x} \mathrm{Cd}_{x} \mathrm{Te} 2 \mathrm{D}$ structures [15].

\subsection{Quantum Hall effect}

One of the most interesting properties of $2 \mathrm{D}$ systems is the quantum Hall effect. The experiments reported previously in literature have demonstrated that in high magnetic fields and at low temperatures the Hall resistivity $\varrho_{x y}$ of a $2 D E G$ exhibits a series of plateaus at the quantized values

$$
\varrho_{x y}=\frac{h}{j e^{2}} \quad(j \text { number of filled Landau levels })
$$

when the external magnetic field or the carrier density is varied. In the present paper we report some high field magnetotransport experiments for an inversion layer on the grain boundary interface of a $\mathrm{p}-\mathrm{Hg}_{1-x} \mathrm{Cd}_{x}$ Te bicrystal using perpendicular magnetic fields up to $15 \mathrm{~T}$. Fig. 7 shows typical data obtained at $1.47 \mathrm{~K}$ for the Hall resistivity component $\varrho_{x y}$ as a function of magnetic field. A similar total carrier concentration $n_{\mathrm{s}} \approx 2 \times 10^{12} \mathrm{~cm}^{-2}$ in the inversion layer and the occupation of three electric subbands as for sample TH364/16 was derived from $\mathrm{SdH}$ oscillation for this sample. In high magnetic fields $(B>10 \mathrm{~T})$ very

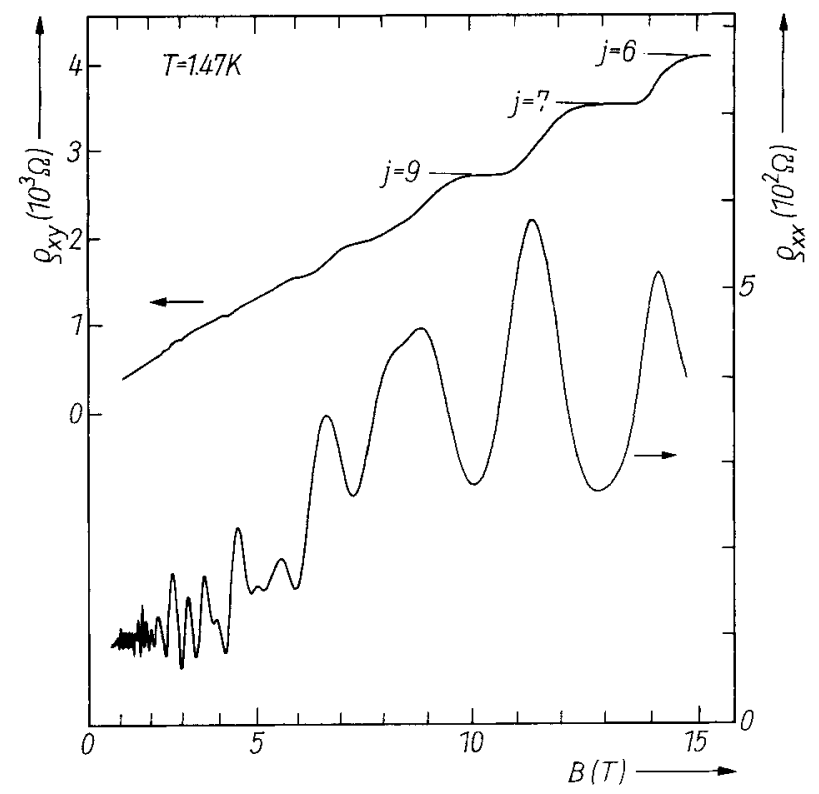

Fig. 7. Diagonal magnetoresistivity $\varrho_{x x}$ and Hall resistivity $\varrho_{x y}$ vs. magnetic field at $T=1.47 \mathrm{~K}$. In high magnetic fields $B>10 \mathrm{~T}$ pronounced spin splitting as well as the integral quantum Hall effect has been observed 
Table 5

\begin{tabular}{clll}
\hline$B(T)$ & $\varrho_{x y}^{\exp }\left(10^{3} \Omega\right)$ & $j=\frac{h / e^{2}}{\varrho_{x y}}$ & $\varrho_{x y}^{\mathrm{th}}=\frac{h}{e^{2} j}$ \\
\hline$>15$ & $4.19 \pm 0.03$ & 6 & 4.302 \\
13.2 & $3.68 \pm 0.03$ & 7 & 3.68 \\
10.3 & $2.80 \pm 0.04$ & 9 & 2.86 \\
\hline
\end{tabular}

pronounced quantum oscillations of $\varrho_{x x}$ and pronounced spin splitting for Landau levels of the lowest electric subband $(i=0)$ are clearly evident. Simultaneously the Hall resistivity $\varrho_{x y}$ shows the characteristic features of the quantized Hall plateaus in finite regions of $B$, weaker plateaus are also apparent in lower fields. Relatively extensive Hall plateaus with quantum numbers $j=6,7$ and 9 are observed, however, the $\varrho_{x x}$ component remains finite and attains values $>100 \Omega$ and the $(j=8)$ plateau is nearly missing. A summary and comparison with the theoretical values are given in Table 5 .

Similar anomalies are obtained qualitatively in InSb grain boundaries [26] and in InGaAs/InP heterojunctions [27]. From a detailed analysis of these results and some numerical calculations it was concluded that the anomalous properties of $\varrho_{x y}$ and $\varrho_{x x}$ can be explained by the existence of several occupied electric subbands in such structures.

\section{Conclusions}

The present results show clearly that a degenerate n-inversion layer exists adjacent to the grain boundary in p-type $\mathrm{Hg}_{1-x} \mathrm{Cd}_{x} \mathrm{Te}$ bicrystals. The rich spectrum of magnetotransport properties ( $\mathrm{SdH}$ oscillations, subband quantization, integral quantum Hall effect, etc.) convincingly demonstrate the quasi-2D character of the electron gas confined in the symmetrical quantum well of the grain boundary interface. The properties are typical of 2D structures involving narrow gap semiconductors with nonparabolic energy bands. A simple theoretical calculation based on the triangular potential well approximation taking into account the nonparabolicity of the dispersion relation is in good agreement with the experimental results. The results show that in suitable circumstances the inversion layer of grain boundaries in narrow gap semiconductors can drastically alter and dominate the electrical transport properties at low temperatures and in high magnetic fields.

The present results represent an interesting contribution both to the physics of $2 \mathrm{D}$ systems and the electronic structure of grain boundaries in narrow gap semiconductors.

\section{Acknowledgements}

The high field experiments have been performed at the International Laboratory for High Magnetic Fields and Low Temperature in Wroclaw (Poland). The authors would like to thank the coworkers of this laboratory for their most valuable technical help and support in the experiments.

The authors also thank Dr. M. Mühlberg for valuable and interesting discussion concerning the growth of mono- and bicrystal samples of $\mathrm{Hg}_{1-x} \mathrm{Cd}_{x} \mathrm{Te}$ alloys and Dr. Hoerstel for helpful discussions. 


\section{References}

[1] W. Scott and R. J. Hager, J. appl. Phys. 42, 803 (1971).

[2] V. I. Ivanov-Omskit, N. N. BerchenKo, and A. I. Elizarov, phys. stat. sol. (a) 103, 11 (1987).

[3] B. M. Vul and E. Y. ZavaritskayA, Zh. eksper. teor. Fiz. 76, 1089 (1979).

[4] S. Uchida, G. Landwehr, and E. Bangert, Solid State Commun. 45, 869 (1983).

[5] R. Herrmann, W. KraAk, G. Nachtwei, and Th. Schurig, phys. stat. sol. (b) 129, 415 (1985).

[6] R. Herrmann, W. KraAk, G. Nachtwei, and G. Worm, Solid State Commun. 52, 843 (1984).

[7] G. Grabecki, T. Dietl, P. Sobkowicz, J. Kossut, and W. Zawadzki, Appl. Phys. Letters 45, 1214 (1984).

[8] G. Grabecki, T. Suski, T. Dietl, T. Skoskiewicz, and M. Glinski, Proc. Internat. Application of High Magnetic Fields in Semiconductors Physics, Würzburg 1986.

[9] R. HerrmanN, W. KraAK, and M. GlińsKi, phys. stat. sol. (b) 125, K85 (1984).

[10] R. Herrmann, W. Kraak, G. Nachtwei, and Th. Schurig, phys. stat. sol. (b) 135, 423 (1985).

[11] W. KraAk, R. Herrmann, G. Nachtwei, and M. Glí́ski, phys. stat. sol. (b) 148, 567 (1988).

[12] W. KraAK, S. Trott, and R. Herrmann, phys. stat. sol. (b) 152, 481 (1989).

[13] J. W. SchOLZ, Thesis, TU München 1983.

[14] W. ZaO, F. Koch, J. Ziegler, and H. Maier, Phys. Rev. B 31, 2416 (1985).

[15] P. Sobkowicz, G. Grabecki, T. Suski, and T. Dietl, Acta Phys. Polon. A75, 39 (1989).

[16] G. Nimtz, B. Schlicht, and R. Dornhaus, Narrow Gap Semiconductors, Springer Tracts in Modern Physics, Vol. 98, 1983.

[17] E. O. Kane, J. Phys. Chem. Solids 1, 249 (1957).

[18] Th. Schurig and R. HerrmanN, phys. stat. sol. (b) 127, K159 (1985).

[19] J. P. Vitton, J. P. Dufour, R. MaCheT, and J. C. Thullier, phys. stat. sol. (b) 120, K53 (1983).

[20] J. Singleton, R. J. Nicholas, F. Nasir, and C. K. Salcor, J. Phys. C 19, 35 (1986).

[21] J. Scholz, F. Koch, J. Ziegler, and H. MaIer, Surface Sci. 142, 447 (1984).

[22] T. I. Der yabina, L. P. Sverev, and V. F. Radantsev, Fiz. Tekh. Poluprov. 17, 2065 (1983).

[23] Y. Takada, K. Aral, and Y. Uemura, Lecture Notes in Physics 152, Springer, 1982 (p. 92).

[24] J. P. Zöllner, G. PAASCH, G. GobsCh, and H. ÜBenSEE, phys. stat. sol. (b) 148, 611 (1988).

[25] R. Herrmann, W. Kraak, S. Handschack, Th. Schurig, D. Kusnick, and B. Schnackenburg, phys. stat. sol. (b) 145, 157 (1988).

[26] R. Herrmann, W. Kraak, and G. Nachtwei, Proc. Internat. Symp. XX. Anniversary Internat. Labor. High Magnetic Fields and Low Temperatures Wroclaw, Wroclaw, May 1988.

[27] Y. Guldner, J. P. Vieren, M. Voos, F. Delahaye, D. Dominguez, J. P. Hirtz, and Razhegy, Phys. Rev. B 33, 3990 (1986). 